\title{
Leishmaniosis felina (L. infantum) en Paraguay. Diagnóstico, tratamiento y evolución
}

\author{
Tintel, M.'; Salvioni, O. ${ }^{1}$; Fraenkel, S..; Marini, R. ${ }^{2}$; Bernal, A. ${ }^{2}$ \\ ${ }^{1}$ Centro para el Desarrollo de la Investigación (CEDIC), Asunción, \\ Paraguay. ${ }^{2}$ Veterinaria Vet Praxis. E-mail: tintelvet $\boldsymbol{a}$ gmail.com
}

\begin{abstract}
Resumen
Tintel, M.; Salvioni, O.; Fraenkel, S.; Marini, R.; Bernal, A.: Leishmaniosis felina (L. infantum) en Paraguay. Diagnóstico, tratamiento y evolución. Rev. Vet. 31: 2, 196-198, 2020. Leishmaniosis es un conjunto de enfermedades zoonóticas causadas por protozoos del género Leishmania sp. Desde tiempo atrás se vienen registrando numerosas infecciones en caninos y en los últimos años ocurrieron casos aislados en felinos. Algunos gatos que cohabitaron en áreas endémicas de leishmaniosis canina contrajeron la enfermedad. Es probable que el sistema inmunitario del felino sea capaz de controlar la contaminación del parásito, ya sea eliminándolo o bien manteniéndolo en un estado crónico subclínico. Se reporta un caso de leishmaniosis en un felino adulto joven, residente en Asunción (Paraguay), cuya sintomatología clínica era inespecífica. Los estudios de laboratorio permitieron identificar la presencia de amastigotes de Leishmania infantum. El paciente fue sometido a tratamiento con respuesta favorable y remisión total de signos clínicos. La identificación del protozooario es determinante para el diagnóstico de esta enfermedad. Si bien la leishmaniosis es menos frecuente en felinos que en caninos, debe incluirse en el diagnóstico diferencial, especialmente en áreas endémicas.
\end{abstract}

Palabras clave: gato, Leishmania infantum, alopurinol, domperidona, sulfadimetoxina.

\begin{abstract}
Tintel, M.; Salvioni, O.; Fraenkel, S.; Marini, R.; Bernal, A.: Feline leishmaniasis (L. infantum) in Paraguay. Diagnose, treatment and evolution. Rev. Vet. 31: 2, 196-198, 2020. Leishmaniasis is a group of zoonotic diseases caused by a protozoa of the genus Leishmania $s p$. There have been numerous cases in canines, and in recent years isolated cases in felines were reported. Therefore, cats that live in endemic areas of leishmaniasis are significantly more likely to be infected. It is probable that feline's immune system is able to control the infection of the parasite, either by eliminating it or by keeping it in a chronic subclinical state. We report a feline case, young adult from Asuncion (Paraguay) with non-specific clinical symptoms. Complementary hematological studies were performed, among them peripheral blood smear and bone marrow puncture, that allowed the identification of the presence of amastigotes of Leishmania sp. L. infantum was identified by molecular techniques. Patient was submitted to treatment, with favorable response and total remission of clinical signs. The identification of the protozoa is determinant for the diagnosis of this disease. Therefore, feline leishmaniasis is less frequent, but not non-existent, and should be included as a differential diagnosis, especially in endemic areas.
\end{abstract}

Key words: cat, Leishmania infantum, allopurinol, domperidone, sulfadimethoxine.

\section{INTRODUCCIÓN}

La leishmaniosis involucra una enfermedad que presenta una gran variedad de manifestaciones clínicas, las cuales difieren tanto en su severidad como en los síntomas producidos.

La diversidad de patologías, que varían desde úlceras cutáneas focalizadas (leishmaniosis cutánea) hasta lesiones en mucosas (leishmaniosis mucocutánea) y órganos internos (leishmaniosis visceral y leishmanio-

Recibido: mayo 2020 / Aceptado: setiembre 2020 sis dérmica post-kala-azar), están determinadas tanto por la especie de Leishmania causante de la infección, como por factores propios del hospedero que se asocian principalmente a la respuesta inmune ${ }^{2}$.

La primera descripción de leishmaniosis en los gatos se remonta a 1912, en Argelia ${ }^{5}$. En los últimos años la frecuencia de casos reportados se ha incrementado ${ }^{7}$ , probablemente por el aumento en la incidencia global de la infección por Leishmania, y por el mayor número de personas que poseen gatos como mascotas ${ }^{6}$

En Paraguay existe poca información sobre la prevalencia e incidencia de esta enfermedad en los gatos. 
El objetivo del presente trabajo fue reportar un caso de leishmaniosis felina en la ciudad de Asunción (Paraguay), incluyendo el modo de identificación de la noxa y la respuesta favorable al tratamiento instaurado.

\section{MATERIAL Y MÉTODOS}

El paciente fue un felino mestizo, macho castrado, de aproximadamente 5 años de edad y $3,8 \mathrm{~kg}$ de peso. El gato acudió a consulta por inapetencia y diarrea negruzca (melena). A la exploración clínica se evidenció desnutrición y problemas dermatológicos como alopecia, engrosamiento de la piel, hiperpigmentación e hiperqueratosis en las zonas auricular, área temporal y cervical inferior.

Inicialmente se procedió a la toma de muestra de sangre para el hemograma. Luego se realizaron los estudios de heces fecales, examen de médula ósea y determinaciones serológicas de enfermedades virales (inmunodeficiencia felina y leucemia felina) ${ }^{1}$.

El protocolo terapéutico utilizado en el paciente fue: alopurinol $(10 \mathrm{mg} / \mathrm{kg}$ cada $12 \mathrm{~h}$ per os durante 2 semanas, luego $15 \mathrm{mg} / \mathrm{kg}$ cada $12 \mathrm{~h}$ per os durante 3 semanas); domperidona ( $1 \mathrm{mg} / \mathrm{kg}$ cada $24 \mathrm{~h}$ per os $\mathrm{du}-$ rante 30 días) y sulfadimetoxina $(20 \mathrm{mg} / \mathrm{kg}$ cada $24 \mathrm{~h}$ per os durante 7 días).

\section{RESULTADOS}

El hemograma (Tabla 1) evidenció disminución del hematocrito, linfocitosis, neutrofilia, eosinofilia y presencia de neutrófilos tóxicos (con corpúsculos de Dōhle).

El examen de materia fecal reveló la presencia de Isospora felis $(++)$. Con relación a los estudios inmunocromatográficos de enfermedades virales, resultaron positivos para inmunodeficiencia felina y negativos para leucemia felina. Las muestras de sangre periférica y médula ósea, revelaron presencia de amastigotes de Leishmania infantum (Figuras 1 y 2).

El aspirado medular se obtuvo empleando el kit comercial GeneJet Genomic DNA Purification Kit (Thermo Scientific ${ }^{\circledR}$ ) y la pureza del material genético se evaluó utilizando un espectrofotómetro $\mathrm{Ds}$-11f $\mathrm{f}+$ (DeNo$\left.v i x^{(\mathbb{R}}\right)$, siguiendo las instrucciones del fabricante. Para confirmar la presencia de Leishmania, fue amplificado un fragmento del gen espaciador intergénico ribosomal (rRNA its-1) utilizando los iniciadores LSgitsl-F1 y LSgits1-R1. Los primers amplifican fragmentos de 220 a $275 \mathrm{pb}$ de longitud dependiendo de la especie.

Cada reacción en cadena de la polimerasa $(P C R)$ fue realizada en un volumen final de $20 \mu \mathrm{L}$, utilizando $10 \mu \mathrm{L}$ del mix (2x hrm PCR Master Mix-Qiagen ${ }^{\mathbb{1}}$ ) a una concentración final de $50 \mathrm{ng} / \mu \mathrm{L}$, y ambos iniciadores a una concentración final de $0,7 \mu \mathrm{M}$. Las reacciones fueron realizadas utilizando un termociclador en tiempo real (Rotor-Gene 6000 Qiagen $^{\circledR}$ ).

La técnica PCR-hrm fue previamente puesta a punto en el laboratorio utilizando controles positivos de cepas de referencia de $L$. infantum, L. amazonensis y L. brazi-
Tabla 1. Hemograma del paciente felino.

\begin{tabular}{lcc}
\hline prueba & resultado & referencia \\
\hline hemoglobina & $9,2 \mathrm{~g} / \mathrm{dl}$ & $8,0-16,0 \mathrm{~g} / \mathrm{dl}$ \\
hematocrito & $27 \%$ & $28-45 \%$ \\
eritrocitos & $6.139 .000 / \mathrm{mm}^{3}$ & $(5-9,5) \times 10,6 / \mathrm{mm}^{3}$ \\
leucocitos & $29.900 / \mathrm{mm}^{3}$ & $(6-17) \times 10 / \mathrm{mm}^{3}$ \\
neutrófilos B & $0,8 \%\left(2.392 / \mathrm{mm}^{3}\right)$ & $0-2 \%$ \\
neutrófilos S & $54 \%\left(16.146 / \mathrm{mm}^{3}\right)$ & $40-75 \%$ \\
linfocitos & $24 \%\left(7.176 / \mathrm{mm}^{3}\right)$ & $20-50 \%$ \\
monocitos & $0,7 \%\left(2.093 / \mathrm{mm}^{3}\right)$ & $1-12 \%$ \\
eosinófilos & $0,7 \%\left(2.093 / \mathrm{mm}^{3}\right)$ & $1-5 \%$ \\
basófilos & $0 \%\left(0 / \mathrm{mm}^{3}\right)$ & $0-1 \%$ \\
plaquetas & $466.000 / \mathrm{mm}^{3}$ & $(250-500) \times 10^{3} / \mathrm{mm}^{3}$ \\
proteínas T & $7,33 \mathrm{~g} / \mathrm{dl}^{2}$ & $5,5-7,90 \mathrm{~g} / \mathrm{dl}^{3}$ \\
\hline
\end{tabular}

B: en banda; S: segmentados, T: totales.

liensis. Posteriormente fue validada con muestras humanas provenientes de distintas regiones de Paraguay.

La $P C R$ en tiempo real confirmó la presencia de Leishmania sp. Los valores de melting de los controles positivos de Leishmania utilizados (Figura 3), arrojaron los siguientes valores: L. infantum Tm: $81,00 \mathrm{C}^{\circ}$; L. amazonensis Tm: $81,97 \mathrm{C}^{\circ}$ y L. braziliensis Tm: $80,05 \mathrm{C}^{\circ}$. El valor de Tm para la muestra L194AM fue de $80,99 \mathrm{C}^{\circ}$, coincidiendo con el valor del control de $L$. infantum.

A las 2 semanas de iniciado el tratamiento, el paciente pesó $4,3 \mathrm{~kg}$, con evidente mejoría clínica y convalecencia de las lesiones cutáneas.

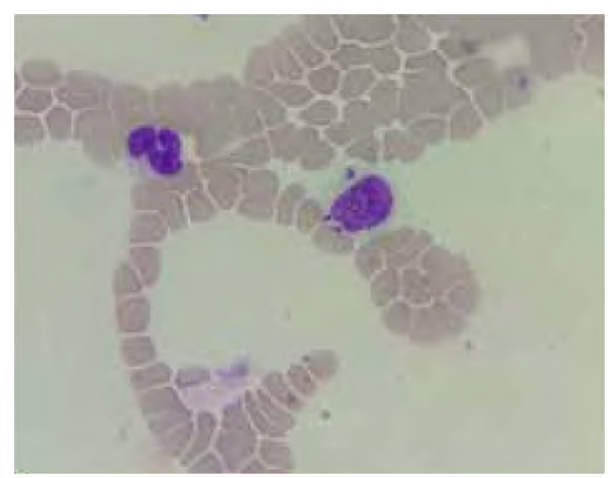

Figura 1. Frotis de sangre periférica revelando un macrófago con amastigotes intracitoplasmáticos de Leishmania infantum (Giemsa 100X).

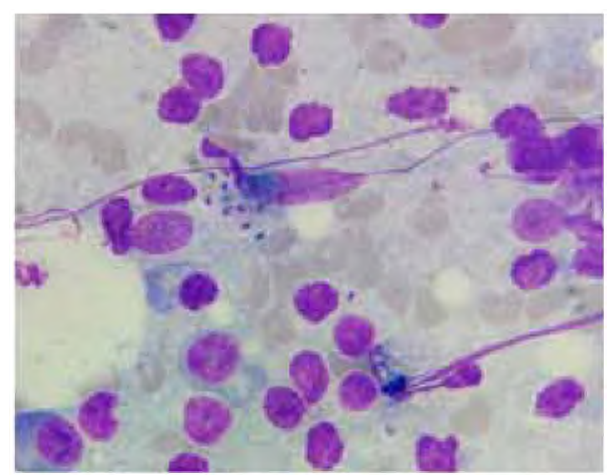

Figura 2. Médula ósea con presencia de amastigotes de Leishmania infantum intra-y extracelulares (Giemsa $100 \mathrm{X}$ ). 


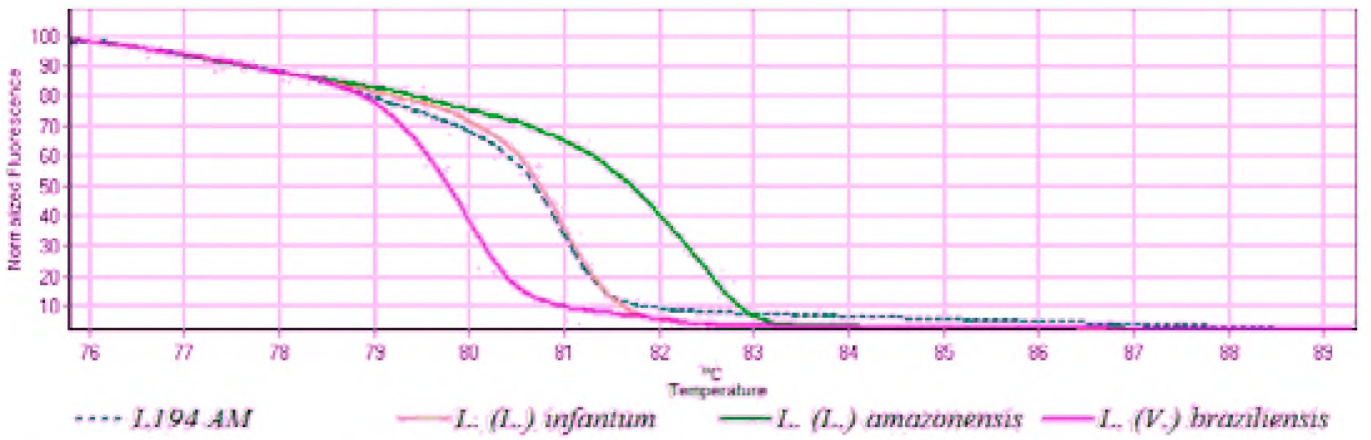

Figura 3. Curva normalizada del fragmento ITS-1. Temperatura de fusión en grados centígrados de los fragmentos de L194AM (izquierda). Hacia la derecha: L. infantum, L. amazonensis y L. braziliensis.

\section{DISCUSIÓN}

Cabe destacar que la mayoría de los reportes de casos en gatos se originaron en zonas endémicas de leishmaniosis canina, situación que coincide con lo descrito en el presente trabajo. A diferencia de otros vertebrados, los gatos podrían asumir un papel activo en la transmisión.

Entre los principales hallazgos que soportan esta hipótesis, pueden citarse el alto porcentaje de gatos con parasitemia o seropositividad halladas en varios estudios en zonas endémicas, la persistencia de la infección aún después de la aparente curación de las lesiones cutáneas, y la preferencia de ciertas especies de flebótomos por alimentarse de la sangre de los gatos ${ }^{3,4}$. Sin embargo, otros autores consideran que el gato se acomoda mejor a la definición de un hospedero accidental ${ }^{1}$

La ausencia de una detección temprana de la leishmaniosis en los gatos de áreas endémicas, puede conllevar un riesgo potencial para la población humana y para los animales de la zona, ya que el felino podría ser un portador asintomático del parásito $\mathrm{y}$ estar disponible para los posibles vectores en el área.

En conclusión, si bien la leishmaniosis es considerada endémica en caninos de Paraguay, no existen evidencias de alta incidencia de casos en felinos. Dicha situación podría atribuirse al sub-diagnóstico o a la menor frecuencia de consultas veterinarias de pacientes felinos ${ }^{6}$.

Resultaría importante que los veterinarios incluyamos a la leishmaniosis como potencial afección de los felinos. El diagnóstico diferencial podrá establecer datos epidemiológicos que evidencien el rol que desempeña el gato en la cadena epidemiológica o como reservorio de tal enfermedad.

\section{REFERENCIAS}

1. Ayllon T et al. 2008. Serologic and molecular evaluation of Leishmania infantum in cats from Central Spain. Ann N Y Acad Sci 1149: 361-364.

2. Beverley SM. 2003. Protozomics: trypanosomatid parasite genetics comes of age. Nat Rev Genet 4: 11-19.

3. Bongiorno G, Habluetzel A, Khoury C, Maroli M. 2003. Host preferences of phlebotomine sand flies at a hypoendemic focus of canine leishmaniasis in central Italy. Acta Trop 88: 109-116.

4. Martin MJ et al. 2007. Infection by Leishmania infantum in cats: epidemiological study in Spain. Vet Parasitol 145: 267-273.

5. Sergent E, Sergent E, Lombard J, Quilichini M. 1912. La leishmaniose à Alger. Infection simultanee d' un enfant, d' un chien, et d' un chat dans la meme habitation. Bull Soc Pathol Exot 5: 93-98.

6. Simoes ML et al. 2005. The susceptibility of domestic cats (Felis catus) to experimental infection with Leishmania braziliensis. Vet Parasitol 127: 199-208.

7. Solano G et al. 2007. Cross-sectional serosurvey of feline leishmaniasis in ecoregions around the Northwestern Mediterranean. Am J Trop Med Hyg 76: 676-678. 